\title{
THE PRESENT POSITION REGARDING THE TREATMENT OF TUBERCULOUS MENINGITIS IN CHILDREN
}

\author{
By R. I. Mackay, M.B., M.R.C.P., D.C.H. \\ Consultant Paediatrician, Hope Hospital, Salford
}

The present position regarding the treatment of tuberculous meningitis in childhood is that parents can be offered a 90 per cent. chance of complete recovery. This transformation is due to the introduction of streptomycin and isonicotinic acid hydrazide. Whereas streptomycin first changed a uniformly fatal disease to one with a mortality of 70 per cent., isoniazid has made it possible to press home the advantage so that 95 per cent. of early cases recover. A long distressing illness has become short. Treatment in the later stages can be continued at home. Tuberculous meningitis can now be considered in similar terms to pyogenic meningitis.

This progress has been illustrated by the experience of Professor Debré1 who recently reviewed groups of children treated by different routines.

1947-Short interrupted courses of streptomycin : 93 cases; 66 deaths; 29 per cent. survival.

1952-Streptomycin continuously for long periods. Intrathecal treatment for many months: 309 cases; 124 deaths; 60 per cent. survival.

1952-54-Streptomycin as above + Para-amino salicylic acid + isoniazid: 220 cases; 39 deaths; 82.5 per cent. survival.

Further analysis of the third series showed a survival rate of 95 per cent. in children over five years of age and 76 per cent. in children between two and five years. Infants still suffer a mortality of 35.5 per cent. Debré quotes other workers (Torres Gost; Ragazzini; Guisti and Cocchi; Choremis; and Fouquet) all of whom have achieved similar survival rates using isoniazid with streptomycin. Lorber ${ }^{2}$ quotes a survival rate of 95 per cent. of 20 children treated in Sheffield in 1954 and a rate of 85.5 per cent. of 69 children treated during 1952-54. In another paper Debré ${ }^{3}$ further demonstrates the improved results obtained by the use of isoniazid from the start of treatment. The survival rate in such cases was 96.3 per cent. compared with 80 per cent. in children for whom isoniazid was an addition to a primary course of streptomycin and PAS.

\section{Isoniazid}

Such is the importance of isoniazid that a review of some of the relevant facts is necessary. Bacterial resistance to isoniazid is rare. Noufflard and Raynaud (quoted by Debré ${ }^{3}$ ) found that only one out of $7 \mathrm{I}$ cultures of $M$. tuberculosis contained organisms resistant to isoniazid and that this culture was not resistant to streptomycin. In a larger series no isoniazid-resistant culture was found among 219 investigations whereas 4 out of I59 were resistant to streptomycin (Debrét). In a report on the treatment of tuberculosis by the Medical Research Council ${ }^{5}$ the development of isoniazid resistance was observed in patients with pulmonary tuberculosis. Sixty-two per cent. of patients excreted organisms resistant to isoniazid after three months' treatment. When streptomycin and isoniazid were combined the corresponding figure was I I per cent.

Isoniazid diffuses readily into all tissues, across the cell membrane and notably across the 'bloodbrain barrier.' Fletcher ${ }^{\circ}$ showed that bacteriostatic levels of isoniazid could be achieved in the cerebrospinal fluid by an oral dose of ro $\mathrm{mg}$. per kg. At the same time he was careful to state that this does not prove an adequate concentration in the brain. Noufflard and Raynaud ${ }^{3}$ also estimated the isoniazid levels in serum and cerebrospinal fluid. A level of 5 to $8 \gamma$ per ml. was obtained in serum two hours after an oral dose of $5 \mathrm{mg}$. per $\mathrm{kg}$. and the equivalent level in the CSF was 2 to $4 \gamma$ per $\mathrm{ml}$. The cultures under examination at the time was sensitive to $0.06 r$ per ml. of isoniazid. This work suggests that oral medication with isoniazid is likely to be effective in tuberculous meningitis. Permeability of the 'blood-brain barrier' to streptomycin, bromide and radiosodium has been shown to be increased in tuberculous meningitis though permeability 
to radiosodium is reduced in obstructive hydrocephalus (Planiol ${ }^{7}$ ).

Most significant is the response of the miliary tubercle to isoniazid. Ritchie et al. ${ }^{8}$ observed that death in cases of tuberculous meningitis treated with isoniazid was due to cerebral vascular accidents and ischaemic necrosis of the brain. In cases not treated with isoniazid, hydrocephalus with organizing basal exudate were the main features. They demonstrated that the essential difference lay in the resolution of tubercles and the absorption of caseation when isoniazid was used. This was followed by the minimum of fibrosis. Dick ${ }^{9}$ observed that the combination of streptomycin with isoniazid seemed the most effective treatment in promoting this type of resolution. They attributed this process to the greater diffusibility of isoniazid in the tissues and introduced a fundamental change in the concept of the pathology of tuberculosis.

The above facts have permitted a new approach to treatment. It is agreed that isoniazid is essential from the first days of treatment. Can it now replace streptomycin? Can isoniazid be relied upon when used alone? Does this development render intrathecal treatment superfluous ?

Bachmann and Wechselberg ${ }^{10}$ claimed a survival rate of 72 per cent. in cases treated with isoniazid alone though the rate was 84 per cent. in cases not given intrathecal isoniazid. In the same paper they report the survival of all the patients in a group of seven treated with streptomycin and isoniazid. Ricci et al. ${ }^{11}$ quote a survival rate of 76 per cent. using a dose of ro to $15 \mathrm{mg}$. per $\mathrm{kgm}$. and Colarizi ${ }^{12}$ achieved $8 \mathrm{r}$ per cent. using 20 to 30 mg. per kg. Both teams used intrathecal isoniazid but used no other antibotic. Ferreira and Ferreira ${ }^{13}$ treated 12 cases with isoniazid alone. Five of these patients had had initial courses of streptomycin and PAS. All cases responded to this regime but were reported after only six months had elapsed at which time the majority were still receiving treatment.

Debré $^{1}$ also mentions reports by Ramos, Walter, Heilmeyer and others showing survival rates of 85 to 90 per cent. in small series of cases. Heilmeyer used very large doses of isoniazid-up to $\mathrm{I} .0 \mathrm{gm}$. daily.

It is not yet possible to pronounce that streptomycin is obsolete nor that isoniazid alone can be relied upon to eradicate the disease in all cases. Lorber's results quoted above are better than any so far reported for a series treated without streptomycin. Bachmann and Wechselberg ${ }^{10}$ report Ioo per cent. success with seven patients given streptomycin and isoniazid but only three patients out of nine survived when given intrathecal but not intramuscular streptomycin with isoniazid.
Spies et al. ${ }^{14}$ obtained almost identical survival rates in two small groups comparing isoniazid alone with streptomycin plus isoniazid but followed the patients for only six months. Debré now recommends isoniazid combined with PAS for early cases without impairment of consciousness but has not yet reported any results of such a regime. It would seem reasonable to retain streptomycin for advanced cases and others with a poor prognosis.

\section{Is Intrathecal Treatment Essential ?}

With increased confidence following the introduction of isoniazid many paediatricians have sought to abandon intrathecal treatment. In a few reports improvement has been noted in seriously ill patients from the time intrathecal medication was stopped. Already many experienced workers have reduced the number of intrathecal injections given to their patients. Debré $^{3}$ at first reduced the period of daily injections to one month. Later he advised that intrathecal administration was necessary only until the patient showed improvement; that is, an improvement in the level of consciousness. In some patients this was less than one week. Lorber $^{2}$ reported a reduction in the number of injections from 90 to a minimum of 25. Daily injections are given for a week followed by three injections each week, the average number in this series being 32 . It is in this series that a survival rate of 95 per cent. was achieved. Ginabreda ${ }^{15}$ quotes a survival rate of 82.6 per cent of 46 cases given intrathecal streptomycin for the first month of treatment whereas only 70 per cent. of 20 children survived when intrathecal treatment was omitted.

Petényi ${ }^{16}$ recently reported a comparison of three series treated by different routines. Ninetyfour patients were treated with streptomycin only in large doses (up to $1.5 \mathrm{gm}$. daily). Of these 13 died (86.2 per cent. survival). Using streptomycin with isoniazid, 88 per cent. of 95 patients survived when given daily medication and in a series of 3 I patients 93 per cent. survived when treatment was given every second or third day. No child was given intrathecal treatment and it was stated in this report that 22 per cent. of the children were younger than two years and 60 per cent. were admitted 'in a paralytic state.' Bulkeley $^{17}$ claimed a survival rate of 93.5 per cent. in 31 children who were treated with corticotrophin. Improvement was so encouraging that the majority of these cases had no intrathecal treatment but eight had had an initial course of intrathecal streptomycin. An earlier group given intrathecal treatment showed a 90 per cent. survival rate. 
Waddell $^{18}$ compared a group of 19 patients given intrathecal treatment of whom I I died. Six children were under 5 years of age (four children less than 2 years) of whom four survived. Five of the six were early cases and the sixth was moribund on admission. The author ${ }^{19}$ has reviewed a series of one hundred consecutive admissions treated by different techniques. In a group of 29 children given combined antibiotics with intrathecal streptomycin the survival rate was 86.2 per cent. Intrathecal treatment was not used in 38 cases for whom medication was otherwise identical. In this group the surival rate was 8r.6 per cent. Heymann ${ }^{20}$ reporting on 600 coloured South African children treated for tuberculous meningitis observed that in late cases only 26 per cent. of I I I children recovered without intrathecal treatment while 38 per cent. of 162 patients given intrathecal medication survived. However, early cases in the same population showed a recovery rate of 90 per cent. whether given intrathecal treatment or not.

In some centres intrathecal medication of all kinds is now avoided and most workers agree that the number of injections can be reduced with safety. In early cases intrathecal injections are no longer necessary.

\section{Steroid Hormones}

Whereas obstructive hydrocephalus was the most significant feature in fatal cases of tuberculous meningitis before isoniazid was introduced, arteritis, thrombosis and ischaemic necrosis of the brain are now the major causes of death and disability in this disease. Corticotrophin and cortisone have therefore been used extensively on the grounds that these hormones lessen inflammatory reaction, decrease basal exudate and inhibit endarteritis.

ACTH has been given by various routes and hydrocortisone has also been given into the theca. The report of Bulkeley ${ }^{17}$ quoted above recommended the use of 6 to 25 units of ACTH every six hours. Erichson ${ }^{21}$ recommended ACTH or cortisone for every advanced case from the inception of treatment and quoted 20 cases in which a successful outcome had been achieved. Heymann ${ }^{20}$ observed an improvement of 13 per cent. in survival rate between two groups of native children. Continuous treatment for two or three weeks is recommended with up to roo units of ACTH daily until improvement is observed. Wide spectrum antibiotics are also used to limit secondary infection. Whether early or advanced cases are considered, sequelae are fewer in children treated with ACTH. Other more startling claims have been made on the basis of very small series.

Shane and Riley ${ }^{22}$ demonstrated the effect of cortisone in a small group of cases. Only four children are included but histological evidence from a fatal case suggests that the cellular reaction and exudate in tuberculous meningitis is much reduced when' cortisone is used. This was not due to isoniazid since it was given to only one patient. Benhamon ${ }^{23}$ recommended the use of cortisone in advanced cases - four dramatic cures were obtained in a group of 14 patients with advanced disease whereas all patients in a control group died. In early cases this writer accepts a recovery rate of Ioo per cent. whether or not cortisone is used. Ashby and Grant ${ }^{24}$ were successful with all patients in a small group of six adults while one patient died in a control group of the same number. Nitscke ${ }^{25}$ studied the response of the CSF in patients treated with cortisone and reported a more rapid return to normal in these cases. If administration of cortisone ceased too soon a regression was observed and as a result this treatment was continued for four months.

Intrathecal hydrocortisone was preferred by Wasz Höckert ${ }^{26}$ who improved the survival rate to a significant degree with its use. Of 24 cases four died in a group given hydrocortisone with streptomycin, isoniazid and PAS. Eleven patients died out of a group of 26 when hydrocortisone was not used although the same anti-tuberculous treatment was employed. This series was extended in a further report in 1956 (Wasz Höckert ${ }^{27}$ ) and in the same meeting Choremis ${ }^{28}$ reported results obtained with intrathecal isoniazid with hydrocortisone in place of intrathecal streptomycin. A survival rate of 83.4 per cent. was quoted for the latter 'orthodox' treatment and 88 per cent. recoveries in the hydrocortisone group.

\section{Adjuvant Treatment}

The development of obstruction to the flow of cerebrospinal fluid is now an infrequent complication of the disease. In early cases it is not seen but the problem may still present in more advanced cases. The routine use of intrathecal tuberculin or tuberculoprotein has never arisen and reports of survival rates are not relevant. As a development of earlier studies Smith et al. ${ }^{29}$ demonstrated a reduction of the bromide ratios in serum-CSF studies during the PPD reaction suggesting an increased permeability of the bloodbrain barrier. Lorber includes intrathecal tuberculin in the regime reported in 1956 . Halikowski ${ }^{30}$ suggests the use of intramuscular ACTH at the height of the reaction to PPD. Smith et al. however, noted that cortisone and antihistamines were antagonistic to the tuberculoprotein reaction and presumably limited the efficiency of this treatment. 
On a similar theme some authors have advised the use of BCG during the course of treatment. The analogy of the Kochs phenomenon is invoked to explain improvement following this procedure. A vigorous response to $B C G$ vaccination indicates a favourable prognosis (Ramos and Torrès-Marty ${ }^{31}$ ).

Before any conclusions are drawn from the above reports it must be made clear that comparisons between different papers may be invalid. The susceptibility of different populations to tuberculosis varies. Uncontrolled selection of patients may be due to geographical factors or related to the state of development of medical services. These factors are not always obvious to the reader and may not even be apparent to the authors. Properly controlled trials are few, but satisfactory series matched for all the known variables can only be accumulated by physicians handling a large number of patients.

\section{Summary of Recommended Treatment}

Early Cases. Provided antibiotics are combined a recovery rate of 95 to 100 per cent. can be anticipated. Isoniazid is essential and is usually combined with streptomycin or PAS. It seems unnecessary to use all three substances. Intrathecal treatment is no longer necessary in early cases.

Late Cases (with impairment of consciousness). The saturation of the central nervous system with antibiotic is urgent. This is achieved by large doses and parenteral administration. Combined antibiotics with isoniazid are essential. Whereas many workers still regard intrathecal treatment as essential for this type of case, survival rates of more than 90 per cent. have been quoted in mixed series when intrathecal medication has been avoided. In this respect ACTH and cortisone have proved their value in maintaining good results. The introduction of isoniazid has made the abolition of intrathecal treatment a practical possibility.

\section{Doses of Medicaments in Current Use}

Streptomycin sulphate (or dihydrostreptomycin), $20 \mathrm{mg}$. per lb. per day (45 mg./kg.). Given intramuscularly in 12-hour doses for the first few days in severe cases then as a daily dose. After improvement has been obtained the calculated dose may be given every third day if the volume of the injection can be adjusted to the available muscle mass.

Isoniazid, Io to $15 \mathrm{mg}$. per lb. per day (20 to $30 \mathrm{mg} . / \mathrm{kg}$.). Oral medication in divided doses 8-hourly is known to be effective: In emergency the 12 to 24 -hour dose may be given intramuscularly until some improvement is obtained.

Para amino salicylic acid (or its salts), 0.2 to $0.3 \mathrm{gm}$. per lb. per day ( 0.4 to $0.6 \mathrm{gm} . / \mathrm{kg}$.). Oral administration is preferred, at 4 or 6-hourly intervals.

$A C T H$ or cortisone (ACTH), 5 to 25 units intramuscularly 6-hourly. Cortisone (or its equivalent as prednisolone) up to $150-200 \mathrm{mg}$. per day. Administration is continued for about three weeks or until some improvement is recognized. Wide spectrum antibiotics must be used to cover this period of treatment.

\section{Duration of Treatment}

At least three months of combined treatment is essential-Debré recommends a minimum of four months. After this phase, isoniazid should be given continously (though the dose may be reduced by 30 to 50 per cent.) for at least one year or up to two years in less favourable cases.

The determination of the end point of treatment is not as simple as has been suggested in some reports. The return of the CSF to normal is a phenomenon which varies from patient to patient. Combined treatment may be continued until all the results of the estimations usually performed have returned to the normal range. Nevertheless, many patients have now been successfully treated without repeated studies of the cerebrospinal fluid and the periods mentioned above seem adequate. Where progress is unsatisfactory, examination of the CSF is essential in the diagnosis of the nature of the complication.

The general assessment of the child in the later stages includes a consideration of weight gain, erythrocyte sedimentation rate and chest radiograph according to established practice. Occasionally psychiatric assessment, intelligence testing and audiometry may be relevant to particular problems of readjustment. About 80 per cent. of the survivors now return to normal life without handicap.

Tuberculous meningitis is becoming rare in Great Britain. This is due to a synthesis of all the measures adopted to control tuberculosis. The more effective antibiotic control of the adult patient at home and the application of BCG vaccination have had a profound effect on the health of the child population.

Recent work suggests that the treatment of all cases of primary tuberculosis with isoniazid (alone or with PAS) may be necessary to eliminate disseminated tuberculosis in childhood (Debré4). This view is disputed by other workers.

Apart from preventative measures, early diagnosis offers most hope of further advance in the reduction of mortality from meningitis. In its early stages this disease is still extremely difficult to recognize in the milieu of general practice. Since it is now so rare, it must first be remembered in differential diagnosis. Persistent headache, 
repeated vomiting, fever of unknown cause and alterations of behaviour are features of the onset. Assurance of a negative contact history must not be relied upon in the event of a clinical suspicion. Even brief contact with a known case of tuberculosis must be regarded as of critical significance. The more general use of Mantoux testing in general practice would make a contribution to early diagnosis.

\section{REFERENCES}

I. DEBRE, R. (1955), 'Acquisitions Medicales Récentes,' p.307.

2. LORBER, J. (1956), Brit. med. F., i, 1009.

3. DEBRE, R. (1955), Presse méd., 63, $4 \mathrm{r}$.

4. DEBRE, R. (1956), Eighth International Congress of Paediatrics. Congress of Paediatrics.

5. MEDICAL RESEARCH COUNCIL (1953), Brit. med. f.,

6. FLETCHER, A. P. (1953), Lancet, ii, 694.

7. PLANIOL, T. (1954), Arch. franc. Pédiate., I1, 465.

8. RITCHIE, G. M., TAYLOR, R. M., and DICK, J. C. (1953), Lancet, ii, 419.

9. DICK, J. C. (1955), Ibid., ii, 216.

ı. BACHMANN, K. D., and WECHSELBERG, K. (1955), Much. Med.'Wschr., No. 20.

1 I. RICCI, G., and COPAITICH, T. (1956), Eighth International Congress of Paediatrics.

12. COLARIZI, A. (1956), Eighth International Congress of Paediatrics.
13. FERREIRA, C., and FERREIRA, N. C. (1953), Arch. franc. Pédiat., ro, 901.

14. SPIES, $H$ W. LEPPER M. M. BLATT, N. H., and DOWLING, H. F. (1954), Amer. Rev. Tuberc., 69, 192.

15. GINABREDA, J. M. S., and MARTINEZ, J. A. (1956), Eighth International Congress of Paediatrics.

16. PETÉNYI, G. (1956), Eighth International Congress of Paediatrics.

17. BULKELEY, W. C. M. (1953), Brit. med. F., ii, I1 27.

18. WADDELL, W. W., et al. (1954), Am. F. Dis. Child., 87, 273.

19. MACKAY, R. I. (1956), Eighth International Congress of Paediatrics.

20. HEYMANN, S. (1956), Eighth International Congress of Paediatrics.

21. ERICHSON, K. (1956), Eighth International Congress of Paediatrics.

22. SHANE, S. J., and RILEY, C. (1953), New Engl. f. Med., 249, 829 .

23. BENHAMON, Ed. (1955), Presse méd., II, I I 16.

24. ASHBY, M., and GRANT, H. (1955), Lancet, i, 65.

25. NITSCKE, A. (1956), Eighth International Congress of Paediatrics.

26. WASZ-HÖCKERT, O. (1955), Nord. Med., 53, 426.

27. WASZ-HOCKERT, O. (1956), Eighth International Congress of Paediatrics.

28. CHOREMIS, C. (1956), Eighth International Congress of Paediatrics.

29. SMITH, H. V., TAYLOR, L. M., and HUNTER, G. (1955), f. Neurol. Neurosurg. Psychiat., 18, 237.

30. HALIKOWSKI, B. (1953), Brit. med. F., i, 991 .

3r. RAMOS, A., and TORRES-MARTY, L. (1955), Presse méd., 63, 703 .

\section{LLOYD-LUKE}

\section{Books that enshrine profound thought}

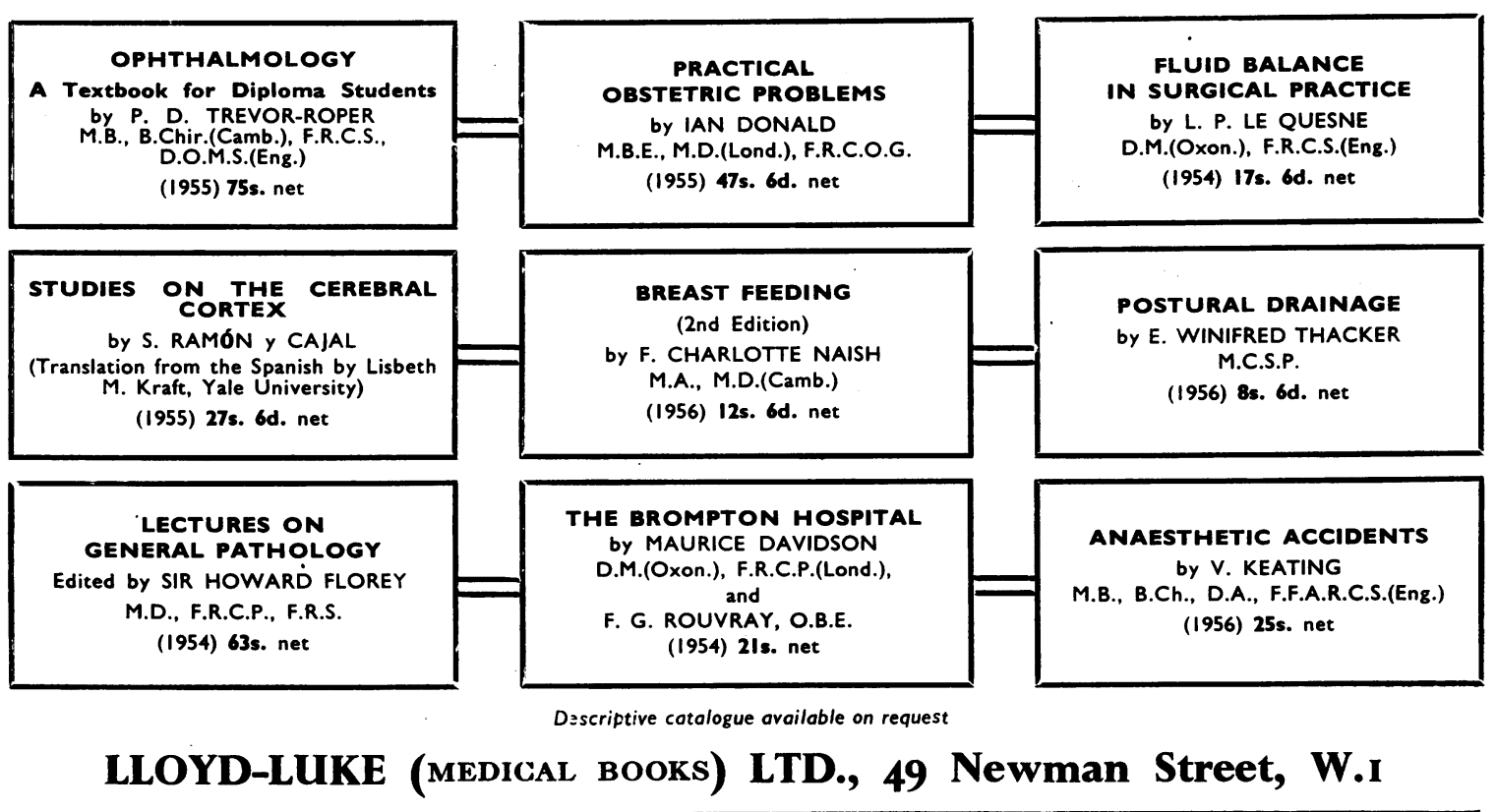

